\title{
Circulating matrix metalloproteinase levels after ventricular septal defect repair in infants
}

\author{
Tim C. McQuinn, MD, ${ }^{a}$ Rachael L. Deardorff, ${ }^{b}$ Rupak Mukherjee, PhD, ${ }^{a, b}$ Anna Greta B. Taylor, MD, ${ }^{c}$ \\ Eric M. Graham, MD, ${ }^{\text {a }}$ Andrew M. Atz, MD, ${ }^{\text {a }}$ Geoffrey A. Forbus, MD, ${ }^{\text {a }}$ Stacia M. DeSantis, PhD, ${ }^{d}$ \\ Jennifer B. Young, ${ }^{\mathrm{a}}$ Robert E. Stroud, MS, ${ }^{\mathrm{b}}$ Fred A. Crawford, MD, ${ }^{\mathrm{b}}$ Scott M. Bradley, MD, \\ Scott T. Reeves, MD, ${ }^{\mathrm{c}}$ and Francis G. Spinale, MD, $\mathrm{PhD}^{\mathrm{b}}$
}

\begin{abstract}
Background: Surgery for congenital heart disease initiates a complex inflammatory response that can influence the postoperative course. However, broad integration of the cytokine and proteolytic cascades (matrix metalloproteinases: MMPs), which may contribute to postoperative outcomes, has not been performed.
\end{abstract}

\begin{abstract}
Methods and Results: Using a low-volume (50-60 $\mu \mathrm{L})$, high-sensitivity, multiplex approach, we serially measured a panel of cytokines (interleukins $2,4,6,8$, and 10, tumor necrosis factor alpha, interleukin $1 \beta$, and granulocyte-macrophage colony stimulating factor) and matrix metalloproteinases (matrix metalloproteinases $2,3,7,8,9,12$, and 13) in patients $(\mathrm{n}=9)$ preoperatively and after repair of ventricular septal defect. Results were correlated with outcomes such as inotropic requirement, oxygenation, and fluid balance. Serial changes in perioperative plasma levels of the cytokines and matrix metalloproteinases exhibited distinct temporal profiles. Plasma levels of interleukins 2, 8, and 10 and matrix metalloproteinase 9 peaked within 4 hours, whereas levels of matrix metalloproteinase 3 and 8 remained elevated at 24 and 48 hours after crossclamp removal. Area-under-the-curve analysis of early cytokine levels were associated with major clinical variables, including inverse correlations between early interleukin 10 levels and cumulative inotrope requirement at 48 hours $(r:-0.85 ; P<.005)$ and late matrix metalloproteinase 7 levels and cumulative fluid balance $(r:-0.90 ; P<.001)$.
\end{abstract}

Conclusions: The unique findings of this study were that serial profiling a large array of cytokines and proteolytic enzymes after surgery for congenital heart disease can provide insight into relationships between changes in bioactive molecules to early postoperative outcomes. Specific patterns of cytokine and matrix metalloproteinase release may hold significance as biomarkers for predicting and managing the postoperative course after surgery for congenital heart disease. (J Thorac Cardiovasc Surg 2010;140:1257-65)

The importance of post-cardiopulmonary bypass (CPB) inflammation in pediatric cardiac surgery is reflected in the many interventions directed at its reduction. Steroid administration, modification of pump circuit surfaces by heparin bonding, ultrafiltration strategies, leukocyte trapping filters, reduced post-CPB oxygen exposure, and monoclonal antibody administration have all been described to modify features of the post-CPB inflammatory response in newborns, infants, and children. ${ }^{1}$ Despite this, a survey of 36 centers performing pediatric $\mathrm{CPB}^{1}$ revealed that no anti-

\footnotetext{
From the Departments of Pediatrics (Cardiology), ${ }^{\mathrm{a}}$ Cardiothoracic Surgery, ${ }^{\mathrm{b}}$ Anesthesia, ${ }^{c}$ and Biostatistics and Epidemiology, ${ }^{\mathrm{d}}$ Medical University of South Carolina and the Ralph H. Johnson VA Medical Center, Charleston, SC.

This work was supported in part by National Institutes of Health grants R01HL059165, HL-057952 (F.G.S.) and a VA Merit Award (F.G.S.), and elements of the work were conducted in a facility constructed with support from NIH C06 RR015455. T.C.M. also received a grant from the National Institutes of Health. Disclosures: None.

Received for publication Sept 28, 2009; revisions received March 18, 2010; accepted for publication May 16, 2010; available ahead of print June 21, 2010.

Address for reprints: Francis G. Spinale, MD, PhD, Cardiothoracic Surgery, STRB Annex, 114 Doughty Street, Charleston, SC 29425 (E-mail: wilburnm@musc.edu). $0022-5223 / \$ 36.00$

Copyright (c) 2010 by The American Association for Thoracic Surgery doi:10.1016/j.jtcvs.2010.05.014
}

inflammatory strategy achieved the level of standard practice in the pediatric CPB population, reflecting absence of convincing data to guide therapies. CPB engenders inflammation through multiple mechanisms, including direct tissue injury, myocardial ischemia/reperfusion, neutrophil/platelet activation from the CPB circuit, and lipopolysaccharide exposure. ${ }^{2}$ The molecular events of inflammation include synthesis and release of cytokines, which in turn induce complex and context-dependent cellular and molecular events, including release of matrix metalloproteinases (MMPs). ${ }^{3}$ The characteristic pleiotropy, redundancy, and complexity of the inflammatory response have complicated efforts to define this process in infants owing to the number of inflammatory biomarkers to consider in relation to relative volume of blood available for sampling. This study was performed to address this issue through the use of high-sensitivity multiplex assays to allow serial measurement of cytokine and MMP levels from repeated small-volume blood sampling in a population of infants undergoing repair of ventricular septal defect (VSD). This study provided the first opportunity to perform a comprehensive cytokine/MMP profile and begin to identify potential relationships between the inflammatory response and clinical outcomes. 


$$
\begin{aligned}
& \text { Abbreviations and Acronyms } \\
& \begin{aligned}
\text { AUC } & =\text { area under the curve } \\
\text { BSA } & =\text { body surface area } \\
\text { CPB } & =\text { cardiopulmonary bypass } \\
\text { GM-CSF } & =\text { granulocyte-macrophage colony } \\
& \text { stimulating factor } \\
\text { IL } & =\text { interleukin } \\
\text { INF- } \gamma & =\text { interferon gamma } \\
\text { MMP } & =\text { matrix metalloproteinase } \\
\text { Th } & =\text { T-helper } \\
\text { TNF } \alpha & =\text { tumor necrosis factor alpha } \\
\text { VSD } & =\text { ventricular septal defect }
\end{aligned}
\end{aligned}
$$

\section{METHODS \\ Patients}

This study was approved by the Medical University of South Carolina Institutional Review Board (HR 16017 and HR 17161). Entry criteria for the VSD group were planned complete surgical repair of the anatomic defect and age from 1 to 9 months. Exclusion criteria were recognized chromosomal anomalies, prior intracardiac surgery, or complex heart disease. Enrollment was from January 2006 through June 2007, and the cardiac surgeons remained constant during this interval (F.A.C., S.M.B.). The conduct of $\mathrm{CPB}$ was performed in identical fashion and aprotinin was used in all cases. Standard nonpulsatile CPB was used, and the circuit was primed with electrolyte solution (PlasmaLyte A; Baxter Healthcare Corporation, Deerfield, Ill) and 1 unit of fresh-frozen plasma. Banked, packed red blood cells were added to achieve a hematocrit value of approximately $28 \%$ to $30 \%$ during $\mathrm{CPB}$. No steroids were administered preoperatively or in the $\mathrm{CPB}$ prime. Moderate $\left(25^{\circ} \mathrm{C}-28^{\circ} \mathrm{C}\right)$ hypothermia was used, and myocardial preservation was obtained with cold blood cardioplegia at 20-minutes intervals. The $\mathrm{pH}$-stat regimen was used during cooling and alpha-stat for rewarming. Modified ultrafiltration was performed after separation from CPB. Protamine was given at a $0.6: 1$ protamine/heparin ratio. Blood product transfusions after CPB were administered as necessary to achieve satisfactory hemostasis and a target hematocrit value of more than $30 \%$. Standard transatrial closure of the VSD was used in all patients. The aprotinin dose consisted of both an intravenous and pump prime load of $240 \mathrm{mg} / \mathrm{m}^{2}$ body surface area (BSA) $\left(1.7 \times 10^{6} \mathrm{kIU} / \mathrm{m}^{2}\right.$ BSA) and a continuous infusion at $56 \mathrm{mg} / \mathrm{m}^{2}$ BSA $/ \mathrm{h}\left(4 \times 10^{5} \mathrm{kIU} / \mathrm{m}^{2}\right.$ $\mathrm{BSA} / \mathrm{h}$ ) until the completion of the primary procedure. For the purposes of providing a referent control range, a group of 10 infants were recruited from patients undergoing ambulatory noncardiac surgery. In this group, a 1-time venous blood collection $(1 \mathrm{~mL})$ was performed at the time of intravenous cannulation.

\section{Clinical Data Collection}

Clinical characteristics such as age, weight, and BSA, as well as z-score for weight, were recorded. Data recorded or calculated during the 48-hour study period included CPB and crossclamp times, arterial blood gases, blood counts, and arterial-alveolar oxygen gradients at 4 hours. Inotrope score was calculated as dopamine $\left(\left[\mu \mathrm{g} \cdot \mathrm{kg}^{-1} \cdot \mathrm{min}^{-1}\right] \times \mathrm{h}\right)+$ epinephrine $\left(\left[\mu \mathrm{g} \cdot \mathrm{kg}^{-1} \cdot \min ^{-1}\right] \times \mathrm{h} \times 100\right)+$ milrinone $\left(\left[\mu \mathrm{g} \cdot \mathrm{kg}^{-1}\right.\right.$. $\left.\min ^{-1}\right] \times \mathrm{h} \times 10$ ). Fluid balance was calculated as total fluid administered (all sources) minus total fluid out (all sources). Inotrope score, fluid balance, and near-infrared spectroscopy were analyzed as cumulative values from time of arrival in the postoperative care unit. In addition, blood product use was recorded during the operative and perioperative periods.

\section{Blood Collection and Processing}

Baseline blood $(1 \mathrm{~mL})$ was collected in the operating room before the incision into a chilled ethylenediaminetetraacetic acid tube. Plasma was isolated by centrifugation, decanted into aliquots, and stored at $-70^{\circ} \mathrm{C}$ until processed for immunoassays. Subsequent $1-\mathrm{mL}$ samples were obtained after modified ultrafiltration and 4, 12, 24, and 48 hours after crossclamp removal and processed identically. Crossclamp removal (onset of myocardial reperfusion injury) was the reference time 0 for postoperative measurements.

\section{Quantitative Measures of Cytokines and MMPs}

Plasma levels of cytokines and MMPs were determined by multiplex suspension array using calibrated and validated combinatorial immunoassays (R\&D Systems, Minneapolis, Minn). For cytokines, measurements were made from undiluted plasma. For MMP analysis, plasma was diluted $1: 10$ for MMP- $3,-7,-8,-12$, and -13 and 1:100 for MMP-2 and -9 . The identification and quantification of the analyte/bead complexes were determined by flow cytometry with dual excitation lasers (the excitation and emission wavelengths are $532 \mathrm{~nm}$ and $575 \mathrm{~nm}$, respectively; Bio-Plex Suspension Array Workstation; Bio-Rad, Hercules, Calif). Each analyte concentration was calculated from an analyte-specific 5-parameter logistic calibration equation (Bio-Plex Manager Software 4.1.1). Average sensitivities for cytokines were $0.3 \mathrm{pg} / \mathrm{mL}$ and $5 \mathrm{pg} / \mathrm{mL}$ for MMP/tissue inhibitors of metalloproteinases. The cytokine and MMP multiplex assays have less than $0.5 \%$ cross-reactivity and interference with the other measured analytes. Plasma values were corrected for hemodilution using simultaneously obtained hematocrit values.

\section{Data Analysis}

Patient demographics and preoperative values for plasma levels of cytokines and MMPs were compared between the control and VSD groups by the Kruskal-Wallis test of medians. Comparisons were withheld if the analyte failed to be detectable in at least $25 \%$ of the samples for each group. For postoperative analysis, 2 area-under-the-curve (AUC) values were derived from the time course of expression for each analyte. Early AUC was determined as the dose $\times$ time product (summation of the areas of individual trapezoids) for each analyte from baseline to the 4-hour post-crossclamp time point. Late AUC was computed in the same fashion for the 4- to 48-hour interval. For AUC determinations, an undetectable level was set at 0 . Correlations between AUCs for each cytokine/MMP and clinical outcomes, as well as between early and late AUCs, were examined by the Spearman correlation analysis. Statistical tests were performed using the Stata software package (Stata Intercooled, v8.0) or SAS (SAS, Inc, Cary, NC).

\section{RESULTS \\ Demographics}

The patients undergoing VSD closure were $4.3 \pm 0.5$ months old, whereas the referent normal control subjects were older $(7.8 \pm 1.3$ months; $P<.05)$, but nevertheless could be used for the purposes of generating a referent normal range for the cytokine and MMP assays. The male/female ratio in the VSD group was 4:5 and similar in the referent control group (5:5). Preoperative white blood counts, platelet counts, and serum measurements of renal function (electrolytes, blood urea nitrogen, and creatinine, not shown) were all within the normal range for the VSD patients. Crossclamp time, CPB time, intubation times, cumulative introtrope score, fluid balance, near infrared spectroscopy, and blood product use for the VSD patients are summarized in Table 1. 
TABLE 1. Intraoperative and perioperative characteristics in subjects with a VSD

\begin{tabular}{lc}
\hline Crossclamp time (min) & $57.2 \pm 5.0$ \\
CPB time (min) & $95.7 \pm 7.4$ \\
Intubation time (h) & $36.2 \pm 3.3$ \\
Inotrope score $\left(\mu \mathrm{g} \cdot \mathrm{kg}^{-1} \cdot \mathrm{min}^{-1} \times \mathrm{h}\right)$ & $126 \pm 34$ \\
Fluid balance $(\mathrm{mL})$ & $-5.3 \pm 12.5$ \\
NIRS $(\%)$ & $66.3 \pm 2.3$ \\
Blood product use & \\
$\quad$ Packed red blood cells (units) & $1(1)$ \\
$\quad$ Fresh frozen plasma (units) & $0(1)$ \\
$\quad$ Cryoprecipitate (units) & $0(0)$ \\
$\quad$ Platelets (units) & $1(0)$ \\
Sample size (n) & 9
\end{tabular}

Times, inotrope score, and fluid balance are presented as mean \pm SEM. Blood product use is presented as median (interquartile range). VSD, Ventricular septal defect; $C P B$, cardiopulmonary bypass; NIRS, near-infrared spectroscopy, average of values recorded in intensive care unit.

\section{Baseline Plasma Levels of Cytokines and MMPs}

Baseline levels for tumor necrosis factor alpha (TNF- $\alpha$ ), the interleukins (ILs), interferon gamma (INF- $\gamma$ ), and granulocyte-macrophage colony stimulating factor (GM$\mathrm{CSF}$ ) for the referent control and VSD groups are summarized in Table 2. IL-1 $\beta$, IL-10, and GM-CSF were detectable, but to a variable degree, in each group. Other cytokines assayed (TNF- $\alpha$, IFN- $\gamma$, IL-2, IL-6, and IL-8) were detectable at baseline in $90 \%$ or more samples. Plasma levels of IL-2 were lower in the VSD group than in referent control values.

Baseline MMP plasma levels for the referent control and VSD groups are shown in Table 2. MMP-12 was not detectable in either group and therefore was excluded from further analysis. Plasma MMP levels at baseline were similar between the groups, with one notable exception in which MMP-13 was detectable in 5 of 9 VSD patients but 1 of 10 controls $\left(P=.033\right.$, Pearson $\left.\chi^{2}\right)$.

\section{Postoperative Cytokine and MMP Measurements}

Using the multiplex array analysis, we constructed a full temporal profile for cytokines (Figures 1 and 2) and for MMPs (Figure 3). These temporal profiles were examined with respect to referent normal control ranges, baseline values, as well as in reference to the intraoperative eventcrossclamp release and completion of modified ultrafiltration. In general, with the exception of IFN and IL-2, cytokine values increased in the early perioperative period. The cytokine IL-6 increased to the greatest relative degree, and IL-10 levels peaked the earliest. An individual response plot for IL-6 as a function of baseline values is shown in Figure 4. All other cytokines with significant elevations peaked at 4 hours after crossclamp, with the exception of IL- $1 \beta$, which was detected in only 3 of 9 subjects at the end of modified filtration, became more regularly detectable at 4,12 , and 24 hours, and returned to undetectable in 7 of 8
TABLE 2. Baseline plasma cytokine and MMP levels in referent control subjects and subjects with VSD

\begin{tabular}{|c|c|c|c|c|c|}
\hline & Control & IQR & VSD & IQR & $\begin{array}{c}P \\
\text { value }\end{array}$ \\
\hline TNF- $\alpha(\mathrm{pg} / \mathrm{mL})$ & $4.25(10)$ & $3.12-5.62$ & $2.87(9)$ & $2.38-3.89$ & .19 \\
\hline IL-2 (pg/mL) & $4.5(10)$ & $3.07-4.68$ & $1.76(8)$ & $1.09-2.64$ & .03 \\
\hline IL-6 (pg/mL) & $0.57(10)$ & $0.49-0.96$ & $0.83(9)$ & $0.49-2.66$ & .59 \\
\hline IL-8 (pg/mL) & $2.68(10)$ & $1.78-5.24$ & $4.98(9)$ & $3.94-5.17$ & .33 \\
\hline IL-10 (pg/mL) & $0.81(6)$ & $0.00-1.44$ & $0.00(4)$ & $0.00-1.03$ & .63 \\
\hline $\mathrm{INF} \gamma(\mathrm{pg} / \mathrm{mL})$ & $1.11(9)$ & $0.33-1.42$ & $0.33(8)$ & $0.17-0.62$ & .13 \\
\hline $\mathrm{GM}-\mathrm{CSF}(\mathrm{pg} / \mathrm{mL})$ & $0.30(5)$ & $0.00-1.00$ & $0.00(3)$ & $0.00-0.39$ & .23 \\
\hline MMP-2 (ng/mL) & $364(10)$ & $328-471$ & $401(9)$ & $293-536$ & .74 \\
\hline MMP-3 (pg/mL) & $1346(10)$ & $821-2079$ & $1080(9)$ & $720-1816$ & .68 \\
\hline MMP-7 (pg/mL) & $2615(10)$ & $2320-3151$ & $2275(9)$ & $1355-2514$ & .29 \\
\hline MMP-8 (pg/mL) & $1033(10)$ & 810-1941 & $1722(9)$ & $1664-2112$ & .41 \\
\hline MMP-9 (ng/mL) & $26(10)$ & $21-48$ & $39(9)$ & $30-44$ & .37 \\
\hline MMP-13 (pg/mL) & $(1)$ & & $85(5)$ & $0-318$ & $.03 *$ \\
\hline \multicolumn{6}{|c|}{$\begin{array}{l}\text { Values are presented as median (number of samples with detectable levels). VSD, Ven- } \\
\text { tricular septal defect; } I Q R \text {, interquartile range; } T N F-\alpha \text {, tumor necrosis factor alpha; } I L \text {, } \\
\text { interleukins; } I N F \gamma \text {, interferon gamma; GM-CSF, granulocyte-macrophage colony } \\
\text { stimulating factor; } M M P \text {, matrix metalloproteinase. } P \text { values from nonparametric } \\
\text { 2-sample Kruskal-Wallis test of medians except for the final value }(*) \text {, which is } \\
\text { Pearson } \chi^{2} \text {. }\end{array}$} \\
\hline
\end{tabular}

samples at 48 hours. No cytokine remained significantly elevated over baseline levels at 48 hours.

With respect to the full array of MMPs, a dynamic and diverse portfolio of changes occurred in the early perioperative period. Plasma levels of MMP-8 and MMP-9 increased in the early post-CPB period, whereas MMP-2 and MMP-7 levels actually fell from baseline values. Plasma MMP-3 levels increased in the later post-CPB time points and did not return to baseline values. All other MMPs changed significantly from baseline at some time point. A representative individual response plot for MMP-9 is presented in Figure 4. Plasma MMP-2 levels were significantly lower than baseline levels in the early postoperative period but returned to baseline levels at 48 hours.

\section{Postoperative Inflammatory Markers and Clinical Outcomes}

We examined the hypothesis that early or late cytokine/ MMP AUCs were linked to specific operative events or postoperative outcomes such as crossclamp time, inotrope score, net fluid balance, and blood product use. Early IL-10 AUC was negatively correlated with cumulative inotrope requirement at 48 hours $(r:-0.85, P<.005)$. Early GM-CSF was negatively correlated with crossclamp time $(r:-0.85$, $P<.005)$. The late MMP-8 AUC was negatively correlated with crossclamp time $(r:-0.87, P<.005)$, as was late MMP9 AUC $(r:-0.73, P<.03)$. Late MMP-7 AUC was negatively correlated with cumulative fluid balance $(r:-0.9, P<.001)$. The early MMP-2 AUC, as well as the late MMP-2 AUC, was negatively associated with packed red blood cell use $(r=-0.88, r=-0.68$, respectively, both $P<.05)$. 

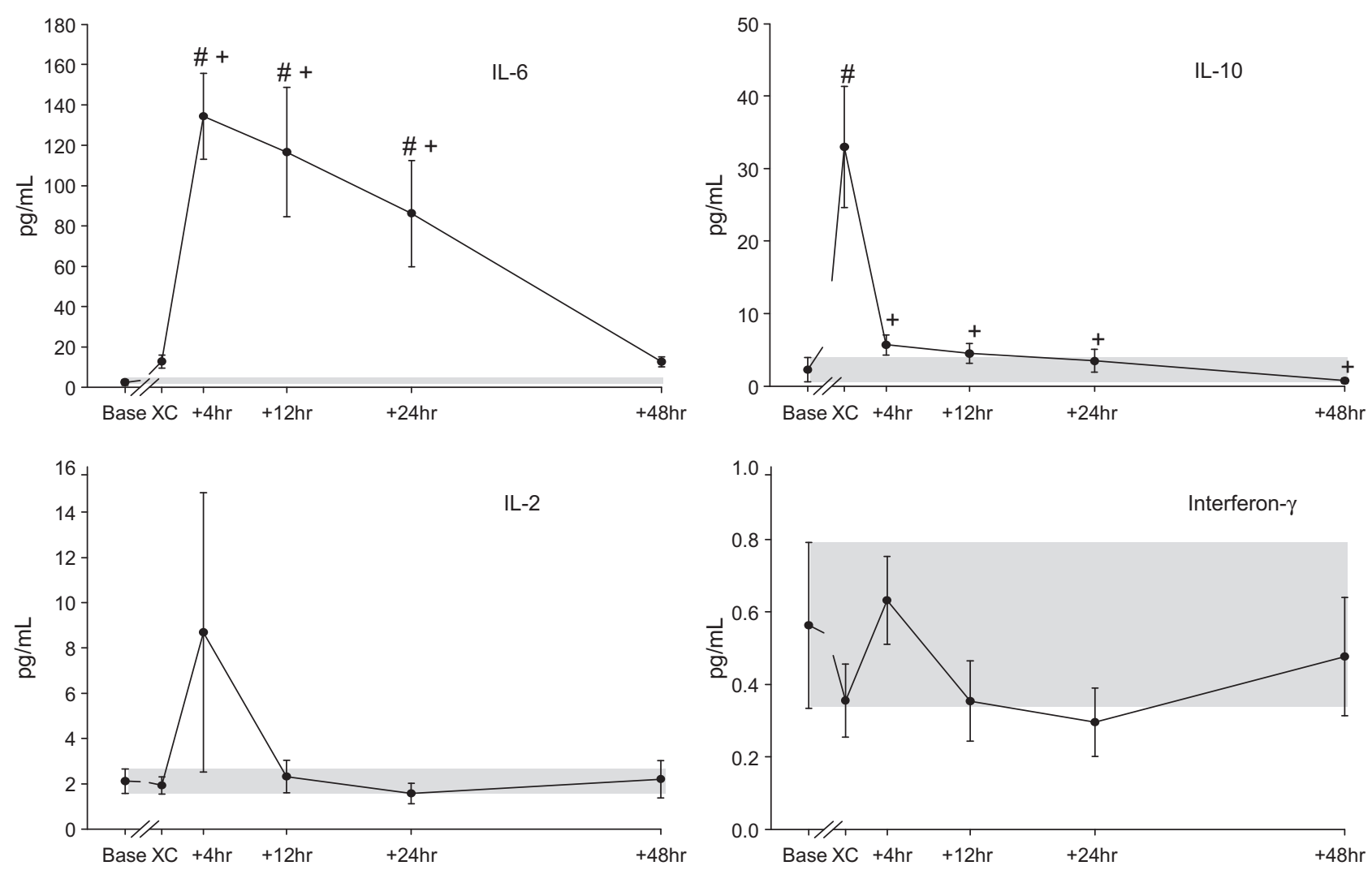

FIGURE 1. Time course for perioperative and postoperative plasma levels ( \pm SEM) of IL-6, IL-10, IL-2, and interferon gamma (INF- $\gamma$ ) in infants undergoing repair of a ventricular septal defect (VSD). The T-helper 1 cytokines (IL-2 and IFN- $\gamma$ ) never increase above baseline values. The shaded bars indicate the range of mean baseline values \pm SEM. Baseline values were obtained before the incision, XC samples were obtained after modified ultrafiltration, and the other time points are measured from crossclamp removal. Note the variation in the $\mathrm{Y}$-axis scale (all in $\mathrm{pg} / \mathrm{mL}$ ). $\# P<.05$ vs baseline values, $+P<.05$ versus values after modified ultrafiltration ( $X C$ values). $I L$, Interleukin.

\section{DISCUSSION}

Although CPB is essential for the conduct of a large number of cardiac surgical procedures, the post-CPB period is associated with a heightened inflammatory response as demonstrated by cellular and noncellular indices of immune activation., ${ }^{2,4}$ This inflammatory response is characterized by the release of cytokines and, through binding to cognate receptors, results in a number of cellular and extracellular events such as cell death and induction of proteolytic pathways. For example, cytokine activation is an upstream pathway for the induction of proteolytic enzymes such as the MMPs. ${ }^{3,5}$ Increased MMP induction in and of itself can lead to significant changes in tissue structure and function and has been demonstrated to play a contributory role in a number of acute and chronic cardiovascular disease states., ${ }^{3,8-12}$ Specifically, MMP induction can directly affect wound healing, alter vascular permeability and reactivity, and contribute to multiorgan dysfunction. ${ }^{8-12}$ Thus, cytokine activation and MMP induction would hold particular relevance in the context of surgical repair of congenital defects requiring CPB. Past studies have focused on a small number of cytokines, at a limited number of time points after pediatric heart surgery requiring $\mathrm{CPB} .{ }^{2,4,7}$ However, full temporal cytokine and MMP plasma profiles have not been simultaneously performed in this patient population previously, and these values related to perioperative outcomes. The unique results of the present study demonstrated that inflammatory cytokine induction occurs after VSD repair in children, which is temporally related to the emergence of a specific MMP profile. Moreover, this specific cytokine and MMP profile was associated with ischemic time (crossclamp time), inotropic requirements, postoperative fluid balance, and blood product use. The present study provided the clinical proof of concept that profiling a large array of relevant bioactive molecules reveals a distinctive signature in infants undergoing surgery for congenital heart disease, which may hold relevance with respect to the underpinning pathophysiologic events that occur in the early postoperative period as well as potential adjunctive use as biomarkers for prognosis and perioperative management. 

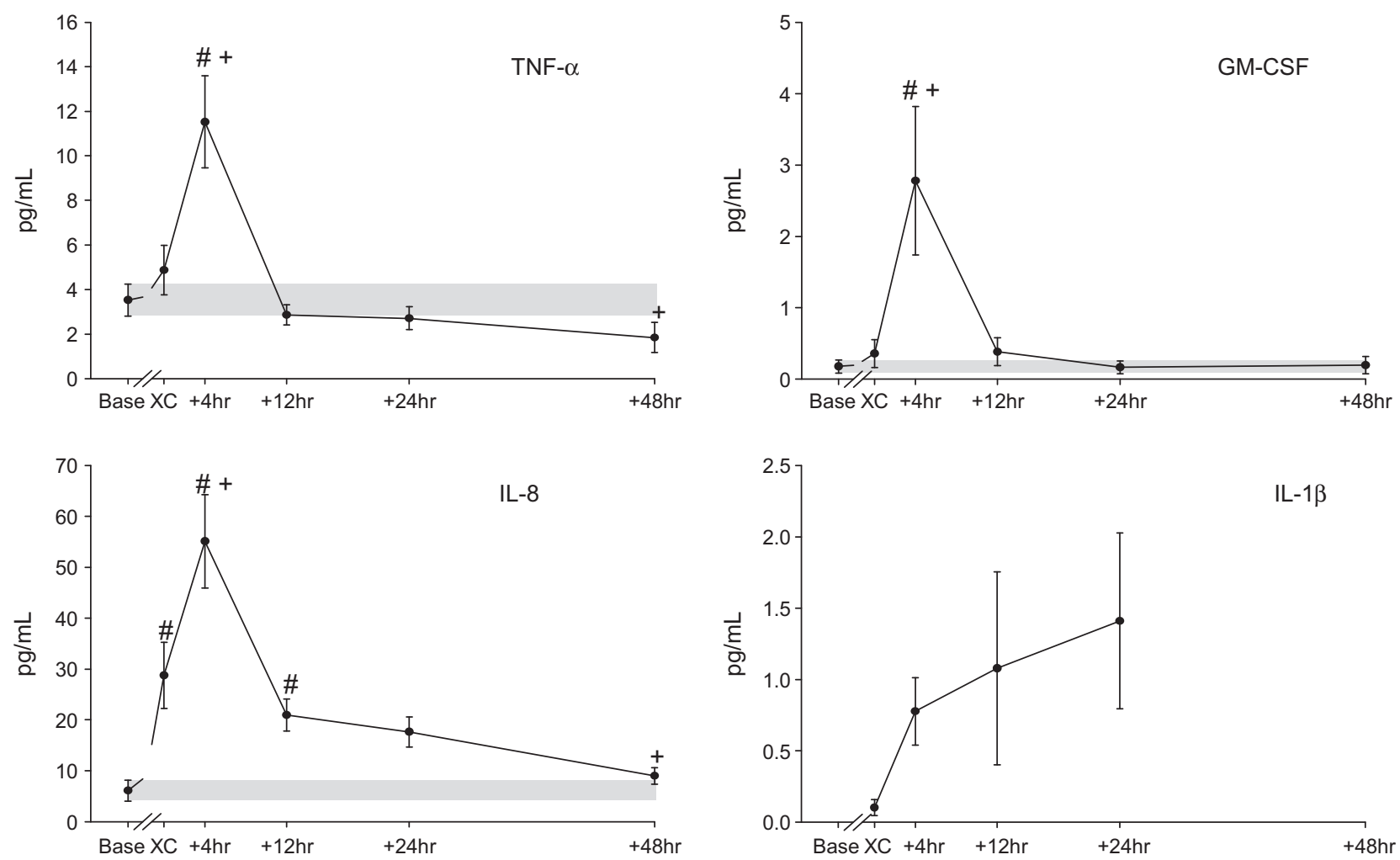

FIGURE 2. Time course for perioperative and postoperative plasma levels of the cytokines TNF- $\alpha$, GM-CSF, IL- 8 , and IL- $1 \beta$ in infants undergoing repair of a ventricular septal defect (VSD). TNF- $\alpha$, GM-CSF, and IL- 8 have similar time courses, peaking at 4 hours and declining rapidly. IL- $1 \beta$ increases from essentially undetectable levels to a peak at 24 hours before returning to generally undetectable levels at 48 hours. The shaded bars indicate the range of mean baseline values \pm SEM. Baseline values were obtained before the incision, XC samples were obtained after modified ultrafiltration, and the other time points are measured from crossclamp removal. Note the variation in the $\mathrm{Y}$-axis scale (all in $\mathrm{pg} / \mathrm{mL}$ ). \#P<.05 versus baseline values, $+P<.05$ versus values after modified ultrafiltration ( $X C$ values). $T N F-\alpha$, Tumor necrosis factor alpha; $G M-C S F$, granulocyte-macrophage colony stimulating factor; $I L$, interleukin.

\section{Temporal Profile of Cytokine Release After VSD Repair}

Past studies that have examined cytokine release, particularly that in surgery for congenital heart disease, have been limited by either cytokine type or time points after CPB. ${ }^{2,4,7,13}$ One likely rate-limiting step in these past studies is that cytokines were performed using a high sample volume immunoassay approach, and therefore blood sample volumes would be problematic. In the present study, a validated high-sensitivity approach was used that provided the ability to measure multiple analytes using a single, small-volume plasma sample. ${ }^{14}$ Through this approach, multiple cytokines could be measured simultaneously, which thereby reduced intrinsic assay variability and allowed for sequential analysis. Moreover, these values were placed in context with referent normal values obtained from children without cardiovascular disease. The outcomes from this analysis demonstrated that a robust increase in certain cytokines occurred immediately after CPB, which included IL-2, IL6 , IL-8, IL-10, and IL-1 $\beta$, TNF- $\alpha$, and GM-CSF. Although the peak levels of IL- 6 and IL-10 that were observed in the present study are consistent with those reported previously after surgery for congenital heart disease, ${ }^{4,7}$ the ability to perform a full cytokine array across time provided some unique insights. First, the pattern of cytokines released is likely reflective of the underlying biology of the immune response in these VSD patients. Specifically, a polarization of cytokine expression occurs early in development, with subsets of lymphocytes such as T-helper 1 cells (Th1) expressing IL-2, TNF- $\alpha$, and INF, whereas T-helper 2 (Th2) cells do not. ${ }^{15}$ There were 3 relevant observations from the present study that would suggest that a Th2 polarization occurred in VSD patients. First, the Th1 cytokine IL-2 values were lower at baseline in VSD infants compared with referent normal values. Second, perioperative values of the Th 2 cytokines IL-10 and IL- 6 were significantly increased. Third, the Th1 cytokines IFN- $\gamma$ and IL- 2 were reduced in the perioperative period. This pattern of mild Th2 polarization has been observed previously in newborns. ${ }^{15}$ This specific cytokine profile remained elevated for a much greater period after CPB than in adults undergoing cardiac surgery. ${ }^{16}$ These findings underscore the fundamental differences in the immune response in newborns undergoing cardiac surgery and that the subsequent inflammatory 

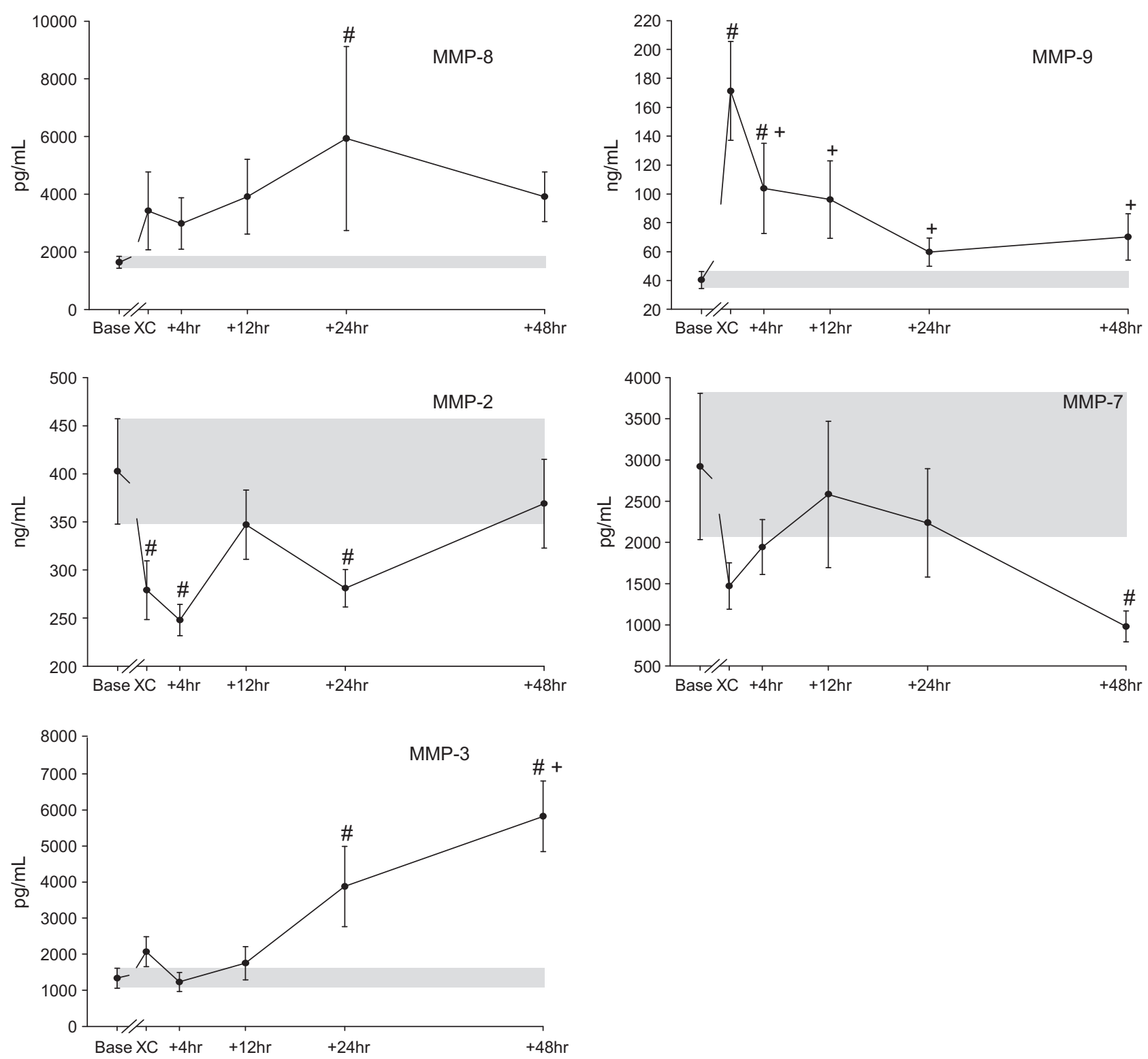

FIGURE 3. Time course for perioperative and postoperative plasma levels of MMP-2, MMP-3, MMP-7, MMP-8, and MMP-9 in infants undergoing repair of a ventricular septal defect (VSD). MMP-2 and MMP-7 values drop below baseline, whereas MMP-8 and MMP-9 values increase with dissimilar time courses. MMP-3 increases steadily through the period from 4 to 48 hours postoperatively. The shaded bars indicate the range of mean baseline values \pm SEM. Baseline values were obtained before the incision, XC samples were obtained after modified ultrafiltration, and the other time points are measured from crossclamp removal. Note the variation in the $\mathrm{Y}$-axis scale (all in $\mathrm{pg} / \mathrm{mL}$ ). $\# P<.05$ versus baseline values, $+P<.05$ versus values after modified ultrafiltration ( $X C$ values). $M M P$, Matrix metalloproteinase.

response may be much different from that which would be anticipated in older children or adults. Thus, profiling the unique cytokine signature that occurs in the perioperative period after pediatric cardiac surgery may hold relevance when developing specific anti-inflammatory strategies for this particularly vulnerable patient population. ${ }^{1}$

Both IL- 6 and IL- 8 are potent proinflammatory cytokines that are chemoattractants for inflammatory cells ${ }^{2,13}$ and remained substantially elevated up to 48 hours after CPB.
Although the present study was not designed or powered to develop prediction models for cytokine profiles and postoperative outcomes, some relative associations could be made. First, heightened release of proinflammatory cytokines such as IL- 6 and IL-8 after surgery for congenital heart disease has been associated previously with pulmonary dysfunction, capillary leak syndrome, and oxidative stress resulting in changes in endothelial and vascular reactivity and function. ${ }^{2,13}$ Second, IL-10, which can be considered 

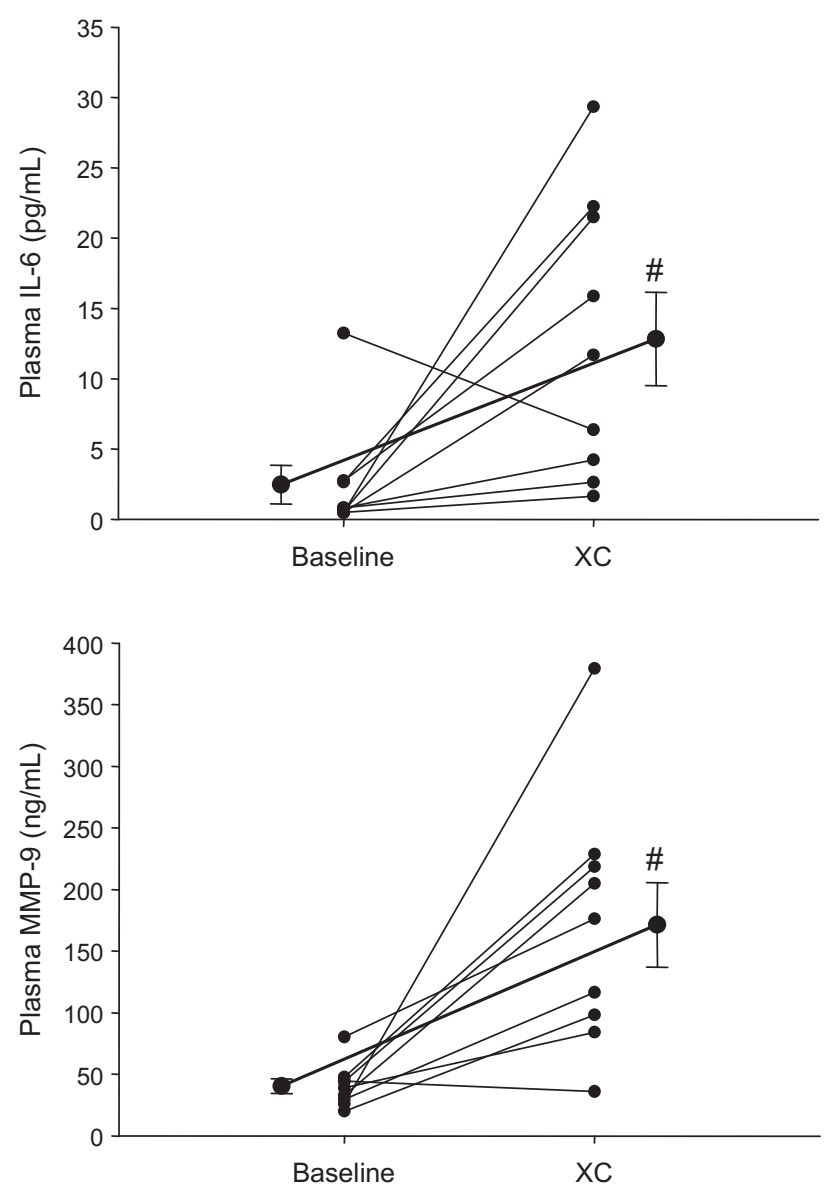

FIGURE 4. Top, Individual response plots for plasma IL-6 levels in the patients undergoing repair of a ventricular septal defect (VSD) at baseline and after crossclamp removal $(X C)$ and modified ultrafiltration. Whereas the relative magnitude varied between patients, IL- 6 levels increased at this early post-CPB time point. Bottom, Individual response plots for plasma MMP-9 levels in the VSD patients at baseline and at XC. Overall, a robust increase in plasma MMP-9 levels occurred at this early post-CPB time point. The darker solid lines indicate the mean baseline and $\mathrm{XC}$ values \pm SEM. $\# P<.05$ versus baseline values. IL, Interleukin; $M M P$, matrix metalloproteinase.

an anti-inflammatory molecule, ${ }^{17}$ increased early in the postCPB period but fell toward baseline values at later time points. Indeed, the present study demonstrated that the relative magnitude of the early release of IL-10 was associated with a decreased inotrope score, suggestive of the potentially protective effects of this specific cytokine. Third, the release of $\mathrm{IL}-1 \beta$ into the circulation is due to cleavage by caspase$1,{ }^{18}$ suggestive of a gradual increase and rapid shutdown of caspase-1-dependent inflammatory pathways in infants after VSD repair. Finally, robust burst of TNF- $\alpha$ release occurred in the VSD patients after CPB, and the myocardium has been shown to be a potent source of this pleotropic cytokine. ${ }^{19}$ In the present study, the relative magnitude of the TNF- $\alpha$ response in comparison to the IL- 6 response after $\mathrm{CPB}$ is characteristic of neonatal monocytes. ${ }^{20}$ One unique bioactive molecule measured in the present study was
GM-CSF, which increased transiently in the early perioperative period and was inversely correlated to crossclamp time. GM-CSF plays a fundamental role in the maturation of the monocyte/macrophage lineage, and transfusion of GMCSF has been used for neonates with neutropenia and sepsis. ${ }^{21}$ However, mechanistic and clinical relationships of changes in GM-CSF in pediatric patients after cardiac surgery will require a much greater sample size and longer follow-up periods.

\section{Temporal Profile of MMP Release After VSD Repair}

A common downstream biological event after cytokine release is the induction and activation of MMPs. Indeed, past studies in adults after cardiac surgery have clearly demonstrated a significant increase in certain MMP types after CPB. ${ }^{5,6}$ Specifically, past studies in adults have identified a robust increase of plasma MMP-8 and -9 after CPB., ${ }^{5,6}$ The present study is the first to examine and compare large portfolios of MMPs in referent normal pediatric subjects, as well as in patients after surgery for congenital heart disease. This approach yielded several important observations. First, detectable plasma MMP-13 levels occurred with greater frequency in VSD patients compared with referent normal subjects. MMP-13 is elevated in the myocardium of patients with left ventricular failure, ${ }^{22}$ and the presence or absence of plasma MMP-13 may hold relevance as to the etiology of the cardiovascular pathophysiologic process that underlies adult patients with left ventricular failure. Second, not all MMP types were uniformly increased in the perioperative period, and the temporal pattern of release appeared to be also different between MMP types. For example, relative MMP-2 and MMP-7 levels actually decreased after CPB. This would imply that there are different regulatory pathways for specific MMP types, which would be operative in children undergoing cardiac surgery. Whether the relative reduction in MMP-2 levels observed in the early perioperative period in the present study was due to a relatively low capacity to upregulate this particular MMP with respect to transcriptional activity, relatively low stores of preformed pro-MMP-2, increased consumption and degradation of this MMP type before spillover into the circulation, or a combination of these factors remains to be established. Potential cardiac sources of MMP-7 include macrophages and cardiomyocytes., ${ }^{3,19}$ Thus, the relative reduction of this specific MMP type may be indicative of the relative immaturity of the immune system and myocardium. Although the mechanisms that underlie the relative reduction in MMP-2 and MMP-7 remain to be established, the present study identified that MMP-2 levels were negatively correlated with packed red blood cell use and MMP-7 levels were negatively correlated with perioperative fluid retention. These observations, albeit associative, underscore the functional diversity of MMPs, whereby some MMP types may play a predominant pathologic role 
whereas other MMP types may contribute to beneficial biological processes.

MMP types that are most associated with an inflammatory process, such as MMP- 8 and MMP-9, increased dramatically in the early post-CPB time points and were temporally related to the release of proinflammatory cytokines such as IL-6, IL-8, and TNF- $\alpha$. Thus, although only associative, this would suggest that the early and robust increase in these cytokines would facilitate the egress of neutrophils and subsequent release of MMP-8 and MMP-9. Indeed, the primary source of MMP-8 is neutrophils and has been consequently termed neutrophil collagenase. The increased levels of these specific MMP types would likely have significant consequences on tissue structure and function in the early post-CPB period. For example, changes in MMP levels can directly affect the wound healing response, and changes in levels of MMP- 8 can directly affect the time course of wound healing. ${ }^{2,10,23}$ Indeed, levels of MMP-3 and MMP-9, both of which changed in the present study, have been associated with wound healing after burn injury in children. ${ }^{12}$ Inasmuch as the relative magnitude of MMP release would likely play a contributory factor in endothelium-matrix interaction and stability, then it is likely that this proteolytic system contributes to the loss of capillary integrity that can often occur after surgery for congenital heart disease. ${ }^{2,10,23}$

\section{LIMITATIONS AND CONCLUSIONS}

Although the present study provided the proof of principle that a large portfolio of cytokines and MMPs can be serially examined in pediatric patients undergoing cardiac surgery, several limitations must be recognized. First, this study was performed in a small number of patients and therefore robust relationships between a specific cytokine/MMP profile to critical outcomes, such as major morbidity and mortality end points, could not be established. Second, the study was performed in the context of a surgical repair of a relatively simple congenital malformation-VSD; therefore, extension of these findings to more complex congenital malformations with respect to cytokine/MMP profiles would be an appropriate future direction. Third, this study was performed in which the nonspecific serine protease inhibitor aprotinin was administered, which in and of itself can potentially alter inflammatory pathways. ${ }^{16,24}$ Inasmuch as aprotinin is no longer available for routine clinical use, then whether and to what degree the cytokine/MMP profile may be altered in the early postoperative period in the absence of this antifibrinolytic therapy remains to be established. Nevertheless, a unique cytokine profile emerged that appears to be $\mathrm{Th} 2$ polarized in infants with VSD, with a dominant IL-6 response, a transient IL-10 response, and an absent IL-4 response. The only cytokine/MMP with an apparent relationship to crossclamp time in this study was GM-CSF, which was suppressed by long CPB times. For the MMPs, the neutrophil-associated proteases MMP-8 and MMP-9 showed marked increases in early post-CPB levels and later sustained elevations that were inversely correlated with crossclamp times. MMP-3 showed a smaller post-CPB spike in expression and a steady late increase that we attribute to wound healing activity. In contrast, circulating levels of MMP-2 and MMP-7 were not elevated above baseline at any time point after CPB. Taken together, these findings suggest that the neutrophil-mediated MMP response after VSD repair in infants is robust (MMP8 and MMP-9) but that early and late significant macrophage, endothelial, and myocyte compartment responses are largely suppressed or absent (MMP-2, MMP-7, anad MMP-12). The mechanisms behind these unique patterns, the dissection of beneficial from deleterious effects, and the benefits of therapeutic manipulation of the inflammatory state engendered by $\mathrm{CPB}$ remain to be determined.

We are grateful to Nikole O'Quinn for her able assistance in the preparation of this manuscript.

\section{References}

1. Checchia PA, Bronicki RA, Costello JM, Nelson DP. Steroid use before pediatric cardiac operations using cardiopulmonary bypass: an international survey of 36 centers. Pediatr Crit Care Med. 2005;6:441-4.

2. Kozik DJ, Tweddell JS. Characterizing the inflammatory response to cardiopulmonary bypass in children. Ann Thorac Surg. 2006;81:S2347-54.

3. Spinale FG. Matrix remodeling and the matrix metalloproteinases: influence on cardiac form and function. Physiol Rev. 2007;87:1285-342.

4. Berdat PA, Eichenberger E, Ebell J, Pfammatter JP, Pavlovic M, Zobrist C, et al. Elimination of proinflammatory cytokines in pediatric cardiac surgery: analysis of ultrafiltration method and filter type. J Thorac Cardiovasc Surg. 2004;127: 1688-96.

5. Lin TC, Li CY, Tsai CS, Ku CH, Wu CT, Wong CS, et al. Neutrophil-mediated secretion and activation of matrix metalloproteinase-9 during cardiac surgery with cardiopulmonary bypass. Anesth Analg. 2005;100:1554-60.

6. Joffs C, Gunasinghe HR, Multani MM, Dorman BH, Kratz JM, Crumbley AJ 3rd, et al. Cardiopulmonary bypass induces the synthesis and release of matrix metalloproteinases. Ann Thorac Surg. 2001;71:1518-23.

7. Madhok AB, Ojamaa K, Haridas V, Parnell VA, Pahwa S, Chowdhury D. Cytokine response in children undergoing surgery for congenital heart disease. Pediatr Cardiol. 2006;27:408-13.

8. Lorente L, Martín MM, Solé-Violán J, Blanquer J, Páramo JA. Matrix metalloproteinases and their inhibitors as biomarkers of severity in sepsis. Crit Care. 2010; $14: 402$

9. Cena JJ, Lalu MM, Cho WJ, Chow AK, Bagdan ML, Daniel EE, et al. Inhibition of matrix metalloproteinase activity in vivo protects against vascular hyporeactivity in endotoxemia. Am J Physiol Heart Circ Physiol. 2010;298:H45-51.

10. Moor AN, Vachon DJ, Gould LJ. Proteolytic activity in wound fluids and tissues derived from chronic venous leg ulcers. Wound Repair Regen. 2009;17:832-9.

11. Pirilä E, Korpi JT, Korkiamäki T, Jahkola T, Gutierrez-Fernandez A, LopezOtin C, et al. Collagenase-2 (MMP-8) and matrilysin-2 (MMP-26) expression in human wounds of different etiologies. Wound Repair Regen. 2007;15:47-57.

12. Dasu MR, Spies M, Barrow RE, Herndon DN. Matrix metalloproteinases and their tissue inhibitors in severely burned children. Wound Repair Regen. 2003; 11:177-80.

13. Brix-Christensen V. The systemic inflammatory response after cardiac surgery with cardiopulmonary bypass in children. Acta Anaesthesiol Scand. 2001;45: 671-9.

14. Ford RL, Mains IM, Hilton EJ, Reeves ST, Stroud RE, Crawford FA Jr, et al. Endothelin-A receptor inhibition after cardiopulmonary bypass: cytokines and receptor activation. Ann Thorac Surg. 2008;86:1576-83.

15. Adkins B, Leclerc C, Marshall-Clarke S. Neonatal adaptive immunity comes of age. Nat Rev Immunol. 2004;4:553-64.

16. Greilich PE, Brouse CF, Whitten CW, Chi L, Dimaio JM, Jessen ME. Antifibrinolytic therapy during cardiopulmonary bypass reduces proinflammatory 
cytokine levels: a randomized, double-blind, placebo-controlled study of epsilonaminocaproic acid and aprotinin. J Thorac Cardiovasc Surg. 2003; 126:1498-503.

17. Couper KN, Blount DG, Riley EM. IL-10: the master regulator of immunity to infection. J Immunol. 2008;180:5771-7.

18. Xing Z, Gauldie J, Cox G, Baumann H, Jordana M, Lei XF, et al. IL-6 is an antiinflammatory cytokine required for controlling local or systemic acute inflammatory responses. J Clin Invest. 1998;101:311-20.

19. Frangogiannis NG, Lindsey ML, Michael LH, Youker KA, Bressler RB, Mendoza LH, et al. Resident cardiac mast cells degranulate and release preformed TNF-alpha, initiating the cytokine cascade in experimental canine myocardial ischemia/reperfusion. Circulation. 1998;98:699-710.

20. Angelone DF, Wessels MR, Coughlin M, Suter EE, Valentini P, Kalish LA, et al. Innate immunity of the human newborn is polarized toward a high ratio of IL-6/ TNF $\alpha$ production in vitro and in vivo. Pediatr Res. 2006;60:205-9.
21. Hercus TR, Thomas D, Guthridge MA, Ekert PG, King-Scott J, Parker MW, et al. The granulocyte-macrophage colony-stimulating factor receptor: linking its structure to cell signaling and its role in disease. Blood. 2009;114: 1289-98.

22. Ahmed SH, Clark LL, Pennington WR, Webb CS, Bonnema DD, Leonardi AH, et al. Matrix metalloproteinases/tissue inhibitors of metalloproteinases: relationship between changes in proteolytic determinants of matrix composition and structural, functional, and clinical manifestations of hypertensive heart disease. Circulation. 2006;113:2089-96.

23. Székely A, Cserép Z, Sápi E, Breuer T, Nagy CA, Vargha P, et al. Risks and predictors of blood transfusion in pediatric patients undergoing open heart operations. Ann Thorac Surg. 2009;87:187-97.

24. Mojcik CF, Levy JH. Aprotinin and the systemic inflammatory response after cardiopulmonary bypass. Ann Thorac Surg. 2001;71:745-54. 